\title{
Modeling of mutants
}

Let $f$ be the successor function of a WT model, and $f_{i}$ the function giving its $i$-th component.

Let $f^{j}$ be the successor function of the same model, with a knock-out mutation of species $j$, and $f_{i}^{j}$ the function yielding its $i$-th component. Let $X$ be a state of the system.

$$
\begin{gathered}
\forall i \neq j, \quad \forall X, \quad f_{i}^{j}(X)=f_{i}(X) \\
f_{j}^{j}(X)=0
\end{gathered}
$$

